\title{
Dissociable Forms of Inhibitory Control within Prefrontal Cortex with an Analog of the Wisconsin Card Sort Test: Restriction to Novel Situations and Independence from "On-Line" Processing
}

\author{
R. Dias, ${ }^{1}$ T. W. Robbins, ${ }^{1}$ and A. C. Roberts ${ }^{2}$ \\ ${ }^{1}$ Department of Experimental Psychology, University of Cambridge, Cambridge, CB2 3EB, United Kingdom, and \\ ${ }^{2}$ Department of Anatomy, University of Cambridge, Cambridge, CB2 3DY, United Kingdom
}

\begin{abstract}
Attentional set-shifting and discrimination reversal are sensitive to prefrontal damage in the marmoset in a manner qualitatively similar to that seen in man and Old World monkeys, respectively (Dias et al., 1996b). Preliminary findings have demonstrated that although lateral but not orbital prefrontal cortex is the critical locus in shifting an attentional set between perceptual dimensions, orbital but not lateral prefrontal cortex is the critical locus in reversing a stimulus-reward association within a particular perceptual dimension (Dias et al., 1996a). The present study presents this analysis in full and extends the results in three main ways by demonstrating that (1) mechanisms of inhibitory control and "on-line" processing are independent within the prefrontal cortex, (2) impairments in inhibitory control induced by prefrontal damage are restricted to novel situations, and (3) those prefrontal areas involved in the suppression of previously established response sets are not involved in the acquisition of such response sets.
\end{abstract}

These findings suggest that inhibitory control is a general process that operates across functionally distinct regions within the prefrontal cortex. Although damage to lateral prefrontal cortex causes a loss of inhibitory control in attentional selection, damage to orbitofrontal cortex causes a loss of inhibitory control in affective processing. These findings provide an explanation for the apparent discrepancy between human and nonhuman primate studies in which disinhibition as measured on the Wisconsin Card Sort Test is associated with dorsolateral prefrontal damage, whereas disinhibition as measured on discrimination reversal is associated with orbitofrontal damage.

Key words: attentional set-shifting; reversal learning; prefrontal cortex; response inhibition; Wisconsin Card Sort Test; working memory
Converging evidence from a diverse range of human and nonhuman primate studies suggests that the prefrontal cortex is critically involved in processes of working memory (Fuster, 1985; Goldman-Rakic,1987; Courtney et al., 1996), behavioral inhibition (Milner, 1964; Mishkin, 1964; Diamond, 1990; Rolls et al., 1994; Knight and Grabowecky, 1995; Dias et al., 1996a,b), and novelty detection (Shallice and Burgess, 1993; Knight and Grabowecky,1995), but how such processes are organized within the prefrontal cortex and whether they occur independently of one another is unclear. From studies in monkeys there is evidence for a global role of the prefrontal cortex in a process that holds representations of stimulus information "on-line" (GoldmanRakic, 1987), an important component of working memory, with independent analysis of visual and spatial information in adjacent prefrontal regions (Wilson et al., 1993). Recently, we provided preliminary evidence for another general process, response inhibition, which operates within different regions of the prefrontal cortex to affect different forms of cognitive processing, even

Received June 16, 1997; revised Aug. 25, 1997; accepted Sept. 11, 1997.

This work was supported by a University Research Fellowship Grant from the Royal Society (A.C.R.) and also by a Programme Grant from the Wellcome Trust (T.W.R., B. J. Everitt, A.C.R., B. J. Sahakian). Dr. R. Dias received a studentship from the Medical Research Council. We thank Dr. R. M. Ridley for supplying the marmosets, Ms. C. Morrison and Ms. H. Sweet for preparation of histological material, and Mr. J. Bashford and Mr. I. Bolton for photographic assistance.

Correspondence should be addressed to Dr. Angela C. Roberts, Department of Anatomy, University of Cambridge, Downing Street, Cambridge, CB2 3DY, UK.

Dr. Dias's present address: School of Psychology, University of Wales, Cardiff, P.O. Box 901, Cardiff CF1 3YG, UK.

Copyright (C) 1997 Society for Neuroscience $0270-6474 / 97 / 179285-13 \$ 05.00 / 0$ within the same modality (Dias et al., 1996a). These findings will now be presented in full together with new data that extend the results in three main ways by demonstrating, first, the independence of the processes of response inhibition and of holding information on-line within the prefrontal cortex; second, that impairments in response inhibition induced by prefrontal damage are restricted to novel situations, and third, that those prefrontal areas involved in the suppression of previously established response sets are not involved in the acquisition of such response sets.

In these experiments, marmosets were trained to make visual discriminations between two compound stimuli, each consisting of a black line superimposed over a blue polygon. For some marmosets the correct response depended on the shape of the black line and for others it depended on the shape of the blue polygon. In Experiment 1 monkeys were trained, preoperatively, to maintain an attentional set (i.e., to respond to a particular perceptual dimension such as the black lines, based on previous experience, over a series of discriminations). Subsequently, the effects of lesions of the lateral or orbital prefrontal cortex were compared on the ability of marmosets to shift their responding at two different levels of response selection, that of stimulus-reward or "affective" associations (visual discrimination reversal) and that of attentional selection for specific perceptual dimensions (attentional set shifting). Performance was studied across repeated shifts to determine the specificity of any disruption to the first occasion that such shifts of responding were required.

In Experiment 2 the marmosets were given no previous experience with the compound stimuli before surgery to establish 
whether the same region of prefrontal cortex that impaired the ability to shift an attentional set in Experiment 1 also contributed to the ability to acquire an attentional set. In addition, Experiment 2 tested explicitly the hypothesis that the deficits in inhibitory control observed in Experiment 1 were independent of deficits in on-line processing.

\section{MATERIALS AND METHODS}

\section{Subjects}

Eighteen common marmosets (Callithrix jacchus), 13 females and 5 males, were used in the present study. Nine marmosets of mean age 15 months were used in Experiment 1, and nine marmosets of mean age 13 months were used in Experiment 2. All monkeys were obtained from the Clinical Research Centre (Harrow, UK) and were housed individually or in sibling pairs. After the daily session of behavioral testing, monkeys were fed $20 \mathrm{gm}$ of MP.E1 primate diet [Special Diet Services (SDS), Withams, Essex, UK], two pieces of carrot, and one piece of apple. The diet was supplemented on weekends with additional fruit, eggs, bread, marmoset jelly (SDS), and peanuts.

\section{Surgery}

Identification of both the lateral and orbital regions of the prefrontal cortex of the marmoset has been described in a previous study (Dias et al., 1996b). In brief, lateral and orbital prefrontal cortex were distinguishable from one another and from surrounding areas on the basis of distinct differences in their cytoarchitectonics. Lateral prefrontal cortex corresponds to Brodmann's area 9, and orbital prefrontal cortex includes Brodmann's areas 10,11, 12, and 13 in his description of the marmoset prefrontal cortex (Brodmann, 1909).

Standardization of stereotaxic coordinates. All marmosets were anesthetized with pentobarbitone $(0.15 \mathrm{ml}$ of a $60 \mathrm{mg} / \mathrm{ml}$ solution, i.p. $)$ and placed in a stereotaxic frame that used a head holder with incisor and zygoma bars specially modified for the marmoset. Given that there is no stereotaxic atlas of the prefrontal cortex in the marmoset and we have found considerable individual variation within the frontal pole, a requirement of the present study was to use infusion coordinates that were tailor-made for each individual marmoset. To achieve this, the thickness of brain tissue was determined at the particular stereotaxic coordinate of anterior-posterior (AP) 17.5, lateral-medial (LM) \pm 1.5 (standardization coordinate) for each marmoset. The thickness was determined by taking a stereotaxic reading as the tip of the infusion syringe pierced the surface of the brain and then again as it touched the base of the brain. If the thickness of tissue was between 5.8 and $6.5 \mathrm{~mm}$, no adjustments were made to the infusion coordinates. However, if the thickness of tissue fell outside of this range, then the standardization coordinate was adjusted accordingly along the anterior-posterior plane until the thickness of tissue fell within this range. For example, if a depth of $5.5 \mathrm{~mm}$ was obtained, then the standardization coordinate had to be moved posteriorly by $0.4 \mathrm{~mm}$ to obtain a thickness of tissue between 5.8 and $6.5 \mathrm{~mm}$. Accordingly, all infusion coordinates would then be adjusted for that particular marmoset, so that rather than using an infusion coordinate of AP 18.5, a coordinate of AP 18.1 would be used.

Excitotoxic lesions of the orbital prefrontal cortex of the marmoset. The orbital region of prefrontal cortex was destroyed by injecting $0.4-0.6$ $\mu \mathrm{l} /$ site of a $0.09 \mathrm{~m}$ solution of quinolinic acid (Sigma, St. Louis, MO) in $0.01 \mathrm{M}$ phosphate buffer, $\mathrm{pH} 7.0$, bilaterally into 10 sites within the prefrontal cortex $(n=3)$. The stereotaxic coordinates that were used are outlined in Table 1 . For all placements, inf usions were made over $100 \mathrm{sec}$ through a stainless steel cannula (30 gauge) attached to a $2 \mu \mathrm{l}$ precision Hamilton sampling syringe. The cannula then remained in place for 4 min before being withdrawn by $1 \mathrm{~mm}$, and it remained at this position for an additional 2 min before being completely removed from the brain.

Excitotoxic lesions of the lateral prefrontal cortex of the marmoset. The lateral region of prefrontal cortex was destroyed by injecting $0.5-1.5$ $\mu \mathrm{l} / \mathrm{site}$ of a $0.09 \mathrm{M}$ solution of quinolinic acid bilaterally into 14 sites within the prefrontal cortex $(n=3)$. The stereotaxic coordinates that were used are presented in Table 1. A $10 \mu$ l Hamilton syringe was used for angled injections, and a $2 \mu \mathrm{l}$ Hamilton syringe was used for upright injections.

All sham-operated control monkeys received infusions of the phosphate buffer vehicle into either the orbital prefrontal cortex $(n=1)$ or the lateral prefrontal cortex $(n=2)$. After surgery, all monkeys that had received the excitotoxin quinolinic acid into the orbital or lateral regions

\begin{tabular}{|c|c|c|c|c|}
\hline \multicolumn{5}{|c|}{ Table 1. Stereotaxic coordinates used to lesion the orbital and lateral } \\
\hline $\mathrm{AP}(\mathrm{mm})$ & $\mathrm{LM}(\mathrm{mm})$ & Angle & $\begin{array}{l}\text { Position of } \\
\text { cannula from } \\
\text { base of skull } \\
(\mathrm{mm})\end{array}$ & $\begin{array}{l}\text { Volume of } \\
\text { quinolinic } \\
\text { acid injected } \\
(\mu \mathrm{l})\end{array}$ \\
\hline \multicolumn{5}{|c|}{ Orbital prefrontal lesion } \\
\hline+16.00 & \pm 4.0 & & 0.8 & 0.4 \\
\hline+16.00 & \pm 2.0 & & 0.7 & 0.5 \\
\hline+16.75 & \pm 3.0 & & 0.7 & 0.6 \\
\hline+17.75 & \pm 2.0 & & 0.7 & 0.6 \\
\hline+18.50 & \pm 2.0 & & 0.7 & 0.5 \\
\hline \multicolumn{5}{|c|}{ Lateral prefrontal lesion } \\
\hline+16.00 & \pm 6.2 & $10^{\circ}$ & 0.9 & 1.0 \\
\hline+16.75 & \pm 5.9 & $8^{\circ}$ & 1.0 & 0.6 \\
\hline+16.75 & \pm 5.9 & $8^{\circ}$ & 1.5 & 0.6 \\
\hline+17.50 & \pm 5.6 & $8^{\circ}$ & 1.0 & 1.0 \\
\hline+18.25 & \pm 5.3 & $8^{\circ}$ & 1.0 & 1.5 \\
\hline+19.00 & \pm 4.6 & $8^{\circ}$ & 0.7 & 0.8 \\
\hline+20.00 & \pm 3.0 & Upright & 1.7 & 0.5 \\
\hline
\end{tabular}

All coordinates were adjusted accordingly, in line with the "depth check" coordinate, as discussed in Materials and Methods.

of prefrontal cortex were administered Valium (Roche, Products, Hertfordshire, UK) $(1.5 \mathrm{mg} / \mathrm{kg}$, i.m.) intermittently over the first $24 \mathrm{hr}$ to suppress any epileptic seizure activity.

\section{Histology}

All monkeys were perfused transcardially with $300 \mathrm{ml} 0.1 \mathrm{M} \mathrm{PBS}, \mathrm{pH} 7.3$, followed by $500 \mathrm{ml} 10 \%$ formalin fixative, administered over $\sim 10 \mathrm{~min}$. The entire brain was then placed in fixative solution overnight before transferral to a $30 \%$ sucrose solution, where it was left for a minimum of $48 \mathrm{hr}$ before histological evaluation. Sections were then cut on a sledge freezing microtome at a thickness of $60 \mu \mathrm{m}$. Every third section was mounted on a gelatin-coated glass microscope slide and stained with cresyl fast violet. A Leitz DMRD microscope was used to view the sections, of which drawings were made with the aid of a drawing tube attachment. Lesioned areas were defined as regions of major neuronal loss often accompanied by marked gliosis. The extent and size of the lesion of each marmoset was then schematized onto drawings of a series of sections depicting the marmoset prefrontal cortex. In addition, the prefrontal cortical lesioned areas were documented photographically using a Zeiss Ultraphot2 (macro). Photomicrographs of the cresyl fast violet-stained coronal sections were taken at both high and low magnification through an intermediate level of the frontal pole of a representative marmoset from the orbital- and lateral-lesioned groups.

\section{Behavioral apparatus}

In the present study both set-shifting ability and reversal learning were measured in a specially designed "hand-testing" apparatus (for a detailed description of the apparatus, see Dias et al., 1996b). In brief, the monkeys sat in a Perspex transport cage, and when one side of it was removed they were able to look through a window with Perspex bars $(21 \times 7.5 \mathrm{~cm})$. On the other side of the window were two wooden boxes $(3 \times 3 \times 7 \mathrm{~cm})$ that were open on only one side and positioned within arm's reach of the monkey on the far right and far left of a shelf $(24 \times 5.5 \mathrm{~cm})$. Attached to the front of each test box was a piece of transparent Perspex behind which the stimulus cards were placed. Two screens, one transparent and one opaque, were placed between the window and the test boxes during the intertrial interval (ITI). At the start of each trial, the opaque screen was removed, leaving the transparent screen in place, and the monkey was allowed to tap the screen immediately in front of one of the two stimuli. A response to one of the stimuli resulted in the removal of the screen, enabling the monkey to turn the chosen box around and remove the reward from within. A response to the other stimulus resulted in the immediate replacement of the opaque screen and no reward. The compound visual stimuli used in all discrimination tasks consisted of black lines (45 mm high) superimposed over blue-filled shapes $(38 \mathrm{~mm}$ high and $38 \mathrm{~mm}$ wide at their broadest point). These stimuli were identical to 
those used in previous set-shifting studies using the hand-testing procedure (Dias et al., 1996a,b), and the only difference from the computerized version of the task used previously in both monkey (Roberts et al., 1992, 1994) and human studies (Owen et al., 1993) was that the lines were black rather than white.

\section{EXPERIMENT 1: A NEUROANATOMICAL ANALYSIS OF ATTENTIONAL SET-SHIFTING AND DISCRIMINATION REVERSAL}

\section{Behavioral methods}

\section{Preoperative testing}

Simple visual discrimination and reversal. All monkeys were trained on a simple visual discrimination (SD1) consisting of either a pair of blue-filled shapes ( $n=4$; "shape" group) (Fig. $1 a)$ or a pair of black lines ( $n=5$; "line" group) presented randomly and simultaneously on two test boxes positioned on the far right and left of the test apparatus. A response to either Shape (S)1 or Line (L) 1 resulted in removal of the transparent screen, allowing the monkey access to a marshmallow (Woolworths) hidden within the test box, whereas an incorrect response to $\mathrm{S} 2$ or $\mathrm{L} 2$ resulted in the replacement of the opaque screen and no reward. A response to either stimulus ended the trial. There was a $5 \mathrm{sec}$ ITI. All subjects received training on the simple visual discrimination for 32 trials/day until they reached a criterion of $90 \%$ correct for three consecutive sessions. Once criterion was attained on the simple visual discrimination, on the following session the reward contingencies were reversed such that the stimulus that had been negatively correlated with reward was now positively correlated with reward and vice versa, i.e., a response to S2 or L2 was now rewarded.

Compound visual discriminations. After successf ul performance on the simple visual discrimination reversal, the second alternative dimension was introduced to form compound stimuli comprising black lines superimposed over blue-filled shapes (CD1) (Fig. $1 b$ ). On any one trial a black line was paired with one of the blue-filled shapes. To succeed, a monkey was required to continue responding to the previously correct stimulus, ignoring the stimuli from the new irrelevant dimension. Two additional compound discriminations [or intradimensional shifts (IDSs)] were presented before surgery, composed of new exemplars from each of the two dimensions (CD2, CD3). For those monkeys trained on blue-filled shapes, shapes remained correlated with reward and one of the two novel shape exemplars was positively associated with reward, whereas for those monkeys trained on black lines, lines remained correlated with reward and one of the two novel black line exemplars was positively associated with reward. As before, stimuli from the second dimension were uncorrelated with reward and therefore remained irrelevant to the discriminations.

All monkeys were then allocated to one of three groups on the basis of their learning scores on the final two compound visual discriminations. They then received infusions of the excitotoxin quinolinic acid into either the orbital (ORB group; $n=3$ ) or lateral (LAT group; $n=3$ ) regions of the prefrontal cortex, or they received sham surgery (control group; $n=3$ ).

\section{Postoperative testing}

Two weeks after surgery, all monkeys were tested for 32 trials/day on a series of visual discriminations, in which advancement to the next discrimination was contingent on reaching a performance level of $90 \%$ correct in two consecutive sessions. The full test design is presented in Table 2, Experiment 1. A summary, however, is provided below.

\section{a) Simple discrimination}

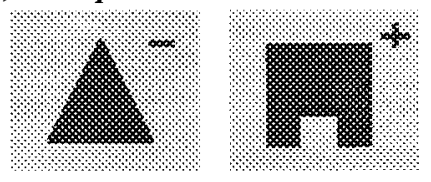

b) Compound discrimination

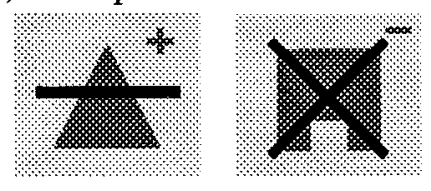

c) Intra-dimensional shift I (IDS)

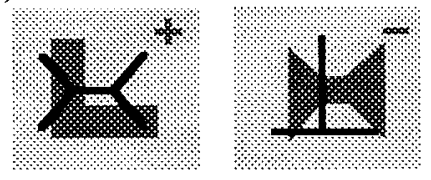

d) Extra-dimensional shift I (EDS1)

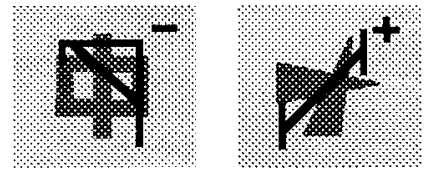

e) Compound reversal I (EDS1R)

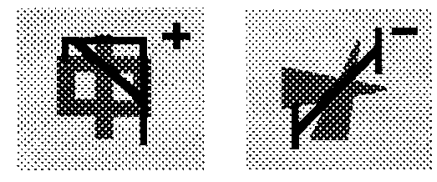

f) Intra-dimensional shift II

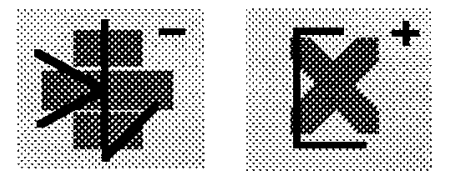

g) Extra-dimensional shift II (EDS2)
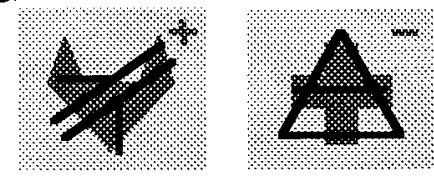

Figure 1. The shape and line exemplars used for the various stages of the attentional set-shifting paradigm. In this example the dimension of "shape" is relevant in all the discriminations except that requiring an EDS $(d)$ and reversal $(e)$ and subsequent IDS II $(f)$. On any one trial of a compound discrimination, a shape exemplar may be paired with one or other of the line exemplars. Correct and incorrect choices are indicated by + and - , respectively. Gray typeface specifies that "shape" is the relevant dimension, whereas black typeface specifies that "line" is the relevant dimension.

(1) Retention of the compound discrimination they had learned immediately before surgery.

(2) A series of three novel compound discriminations or IDSs. Each discrimination (IDS1, IDS2, IDS3) required the monkey to 

Table 2. The shape and line exemplars used for the various stages of the attentional set-shifting
paradigm in Experiments 1 and 2

Discrimination

Exemplars

Experiment 1

Simple

Simple reversal

Series of compound discriminations (CD1,CD2)

Compound discrimination prior to surgery (CD3)

S1- S2+

Surgery

Retention test (CD3)

S5+ S6-

L5 L6

S5+ S6-

L5 L6

Two novel compound discriminations (IDS1,IDS2)

Compound discrimination prior to EDS (IDS3)

S11+ S12-

Probe test

L11 L12

S11+ S12-

L13 L14

Extra-dimensional shift (EDS1)

L15+ L16-

S15 S16

Compound reversal (EDS1R)

L15- L16+

S15 S16

Two novel compound discriminations (IDS4,5) and reversals (IDS4R,5R)

Compound discrimination prior to EDS2 (IDS5R)

L19- L20+

Second extra-dimensional shift (EDS2)

S19 S20

S21+ S22-

L21 L22

Compound reversal (EDS2R)

S21- S22+

L21 L22

Experiment 2

Simple

Simple reversal

$\mathbf{P} 1+\mathbf{P} 2-$

Surgery

Retention test

Compound discrimination (IDS1)

P1- P2+

P1- P2+

$\mathrm{S} 1+\mathrm{S} 2-$

L1 L2

Two novel compound discriminations (IDS2,IDS3)

Compound discrimination prior to compound reversal (IDS4)

S7+ S8-

L7 L8

Compound reversal (IDS4R)

S7- S8+

L7 L8

Compound discrimination (IDS5)

S9- S10+

L9 L10

Compound reversal (IDS5R)

S9+ S10-

L9 L10

S9+ S10-

L11 L12

Extra-dimensional shift (EDS)

L13+ L14-

S13 S14

L13- L14+

Compound reversal (EDSR)

The examples given illustrate the precise sequence of discriminations for those monkeys trained initially on "shapes." $\mathrm{S}$, L, Dimensions of shape and line, respectively; $P$, pattern; $1-22$, individual line and shape exemplars; +, - , reinforcement value of the stimulus. Letters and numbers in bold indicate which dimension is relevant.

learn which of two novel exemplars from the previously relevant dimension was positively correlated with reward (Fig. 1c).

(3) A probe test. After completion of the third IDS (IDS3), the exemplars from the irrelevant dimension were replaced with two novel exemplars, whereas the exemplars from the relevant dimension and the reward contingencies remained the same, i.e., the exemplar from the relevant dimension that had been previously associated with reward continued to be associated with reward. This stage of the task was used to determine that the monkeys were not solving each discrimination on the basis of gestalt images (combining line and shape exemplars to form a compound image) (Roberts et al., 1988). Once the monkeys had reattained 
criterion they were returned to the original compound discrimination (IDS3) before the next stage.

(4) An extradimensional shift (EDS). A new compound discrimination (EDS1) in which one of the two novel exemplars from the previously irrelevant dimension became positively correlated with reward, thus requiring a shift of attentional set from one perceptual dimension of the compound stimulus to another (Fig. 1d).

(5) A compound discrimination reversal. The exemplar from the relevant dimension that had been previously negatively correlated with reward became positively correlated with reward and vice versa (Fig. 1e, EDS1R).

(6) Two novel compound discriminations or IDSs and subsequent reversals (IDS4, IDS4R, IDS5, IDS5R). In the discriminations requiring an IDS, one of the two novel exemplars from the new relevant perceptual dimension was positively correlated with reward (Fig. 1f). In the reversals, the exemplar that had been previously negatively correlated with reward became positively correlated with reward and vice versa. The exemplars from the previously relevant dimension were uncorrelated with reward. This extended series of IDSs and reversals was used to ensure that all monkeys had acquired the new attentional "set" before a second shift.

(7) A second EDS (EDS2). A discrimination comprising novel compound stimuli that required a shift of attentional set from the current relevant dimension back to the other perceptual dimension that had been relevant before the first EDS (Fig. 1g).

(8) A compound discrimination reversal (EDS2R). The exemplar that had been previously negatively correlated with reward in EDS2 became positively correlated with reward and vice versa.

The number of errors that were made before reaching criterion were recorded for each discrimination.

\section{Statistical methods}

All behavioral data were analyzed using the CLR ANOVA statistical package (Clear Lake Research). Whenever the distribution of these variables violated the assumptions made for the ANOVA, an appropriate transformation was used. Planned comparisons were made using simple main effects.

\section{Results}

\section{Histological assessment of lesion}

Both the lateral and orbital prefrontal cortex lesions in the marmoset in this first experiment have been detailed previously (Dias et al., 1996a), including representative photomicrographs and schematic drawings of all the lesioned monkeys. These lesions were similar to those described comprehensively in Experiment 2 of this paper.

\section{Behavioral effects}

Retention and new learning of compound visual discrimination problems. Preoperatively, the monkeys that were scheduled to receive either quinolinic acid or phosphate buffer vehicle into the lateral or orbital regions of prefrontal cortex did not differ in their ability to learn either a simple or compound discrimination $(F<$ 1) (see Table 3 for mean \pm SEM).

Postoperatively, all groups returned to criterion on the first test session (see Table 3 for mean \pm SEM), thus there were no differences in performance of the retention test across the three groups $(F<1)$. Similarly, there was no effect of either lesion on the acquisition of the subsequent series of three novel discriminations or IDS, which required the monkey to maintain attention toward the previously relevant perceptual dimension (see Table 3
Table 3. Mean scores $( \pm$ SEM) for Experiment 1

\begin{tabular}{lrcc} 
& \multicolumn{3}{c}{\begin{tabular}{l} 
Mean number of errors to criterion \pm \\
\cline { 2 - 4 } DEM
\end{tabular}} \\
\cline { 2 - 4 } Discrimination & \multicolumn{1}{c}{ Control } & LAT lesion & ORB lesion \\
\hline All preoperative discriminations & $104.7 \pm 56.1$ & $65.7 \pm 28.6$ & $51.3 \pm 25.0$ \\
Retention test (CD3) & $0.0 \pm 0.0$ & $0.0 \pm 0.0$ & $1.0 \pm 1.0$ \\
Intradimensional shift 1 (IDS1) & $5.7 \pm 5.2$ & $17.0 \pm 5.7$ & $8.0 \pm 3.1$ \\
Intradimensional shift 2 (IDS2) & $11.7 \pm 5.2$ & $5.0 \pm 3.2$ & $10.0 \pm 3.9$ \\
Intradimensional shift 3 (IDS3) & $3.7 \pm 2.5$ & $3.7 \pm 2.5$ & $8.3 \pm 2.1$ \\
Extradimensional shift 1 (EDS1) & $13.3 \pm 1.8$ & $37.0 \pm 6.3^{*}$ & $16.7 \pm 2.2$ \\
Compound reversal 1 (EDS1R) & $16.0 \pm 0.7$ & $18.3 \pm 4.0$ & $41.0 \pm 2.5^{*}$ \\
Intradimensional shift 4 (IDS4) & $1.7 \pm 1.7$ & $2.0 \pm 2.0$ & $0.0 \pm 0.0$ \\
Compound reversal 2 (IDS4R) & $6.3 \pm 2.3$ & $5.7 \pm 2.0$ & $9.3 \pm 3.2$ \\
Intradimensional shift 5 (IDS5) & $0.0 \pm 0.0$ & $0.0 \pm 0.0$ & $0.0 \pm 0.0$ \\
Compound reversal 3 (IDS5R) & $5.3 \pm 0.4$ & $5.7 \pm 2.0$ & $5.3 \pm 3.6$ \\
Extradimensional shift 2 (EDS2) & $6.7 \pm 1.8$ & $7.7 \pm 1.1$ & $5.7 \pm 1.1$ \\
Compound reversal 4 (EDS2R) & $7.7 \pm 2.9$ & $5.0 \pm 3.1$ & $7.3 \pm 1.8$ \\
\hline
\end{tabular}

${ }^{*} p<0.001$.

for mean \pm SEM). ANOVA of the square root transformed data for all three discriminations revealed that there were no effects of Lesion $(F<1)$ or Discrimination $(F<1)$ and that the Lesion $\times$ Discrimination interaction did not reach the $5 \%$ level of significance $\left(F_{(4,12)}=2.81 ; p=0.074\right)$.

Probe test. The introduction of novel exemplars of the irrelevant dimension had no effect on performance in control, lateral prefrontal, or orbital prefrontal lesioned monkeys; in all three groups the monkeys continued to respond to the previously correct exemplar at the $90 \%$ correct criterion level. A comparison of the errors made on the previous test session when all monkeys were performing at the $90 \%$ criterion level revealed no effect of the probe $(F<1)$ or the lesion $(F<1)$, and no interaction of the lesion with the probe $(F<1)$.

$E D S$. All monkeys took longer to acquire the discrimination requiring an EDS compared with the immediately preceding discrimination requiring an IDS (Fig. $2 A$ ), confirming that all monkeys had developed an attentional set and therefore were required to shift attentional set at the EDS stage of the test. However, although the performance of monkeys with lesions of the lateral prefrontal cortex was equivalent to both controls and monkeys with lesions of the orbital prefrontal cortex on the discrimination requiring an IDS, their performance was significantly inferior to both of the other groups on the discrimination requiring an EDS (Fig. $2 A$ ). ANOVA of the total errors to reach criterion on the EDS and the preceding IDS showed a main effect of Shift $\left(F_{(1,6)}=143.73 ; p<0.001\right)$ and a significant Lesion $\times$ Shift interaction $\left(F_{(2,6)}=32.37 ; p<0.001\right)$. Further analysis of the simple main effects showed that although all marmosets regardless of their surgery exhibited superior IDS performance over their EDS performance, those marmosets with lesions specific to the lateral prefrontal cortex were significantly impaired at the EDS stage of the task, making three times as many errors as either orbital prefrontal lesioned or control marmosets before reaching criterion $\left(F_{(2,9)}=23.14 ; p<0.001\right)$.

It is difficult to determine whether the marmosets with lesions of the lateral prefrontal cortex were impaired at the extradimensional stage of the task because they perseverated to the previously relevant dimension. To demonstrate perseveration of an attentional set, it is necessary to demonstrate that monkeys continue to respond to one of the stimulus features, i.e., blue poly- 
(A)

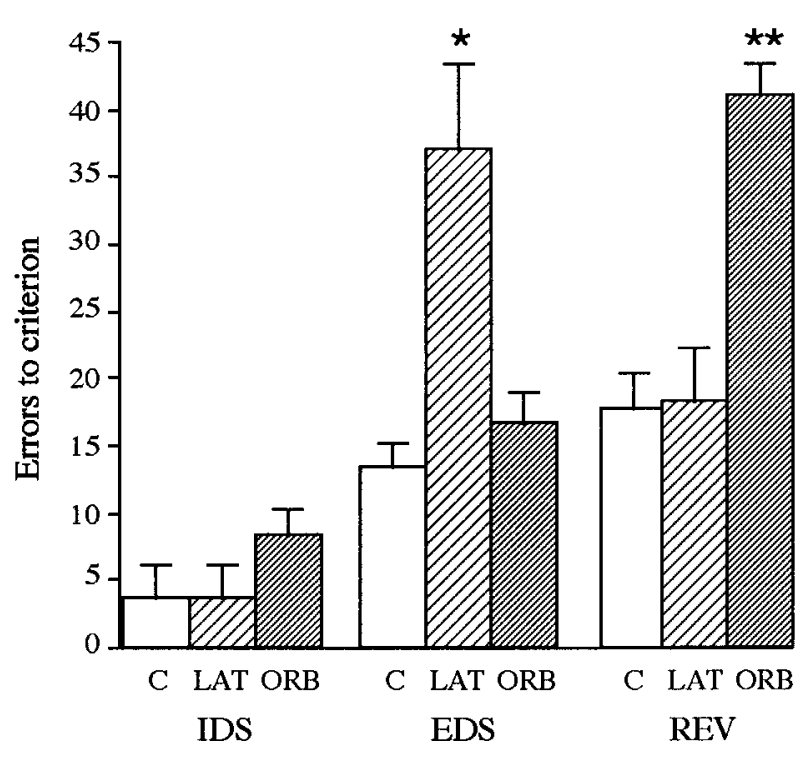

(B)

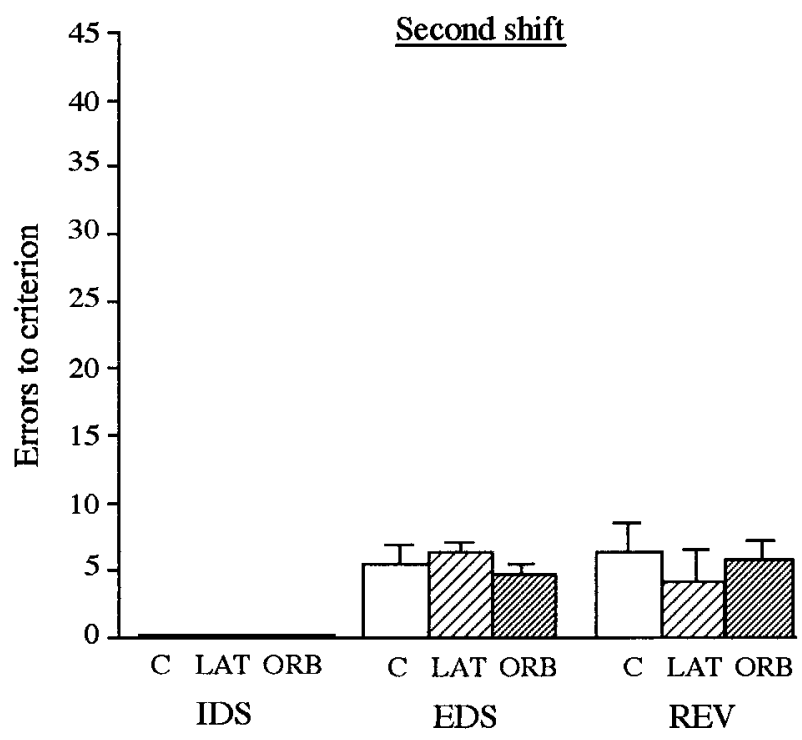

Figure 2. $A$, Mean number of errors ( \pm SEM) to reach criterion on a visual discrimination that requires an IDS (third discrimination of the series of three that were presented postoperatively), an EDS, and a reversal, EDSR, in monkeys that received excitotoxic lesions of either the lateral $(L A T)(n=3)$ (pale hatched bars) or orbital prefrontal cortex $(O R B)(n=3)$ (dark hatched bars) or a sham operation $(n=3)$ (open bars). $B$, Mean number of errors $( \pm$ SEM) to reach criterion on subsequent visual discriminations that require an intradimensional shift (IDS), an extradimensional shift $(E D S)$, and a reversal $(R E V) .{ }^{*}$ Lateral prefrontal lesioned group differed significantly from controls and orbital prefrontal lesioned group; $p<0.001$. ** Orbital prefrontal lesioned group differed significantly from controls and lateral prefrontal lesioned group; $p<0.001$.

gons but not black lines, over many trials. However, because on any one trial each of the two compound stimuli are composed of a blue polygon paired with one of the black lines, it is not possible unambiguously to determine from a single trial whether the monkey is responding to blue polygons or black lines. Consequently, performance must be monitored over multiple trials, and then perseveration can be characterized only if the monkey chooses consistently over a series of trials one of the exemplars from the previously relevant dimension. Such behavior has been displayed, to varying degrees, by monkeys in our previous setshifting studies (Roberts et al., 1992, 1994), and in the former of these the extent of this perseveration was shown to correlate positively with their overall performance on the EDS. In this experiment, two out of the three sham-operated monkeys responded repeatedly (five or six consecutive responses; $p<0.05$ ) to one of the exemplars from the previously relevant dimension at the start of the EDS. The same was true for all three monkeys that received an orbital prefrontal lesion and two of the three monkeys that received a lateral prefrontal lesion. Similar perseverative patterns were not seen at the start of the immediately preceding IDS, showing that all monkeys were indeed attending to the previously relevant dimension at the start of the EDS. However, there was no obvious difference between the three groups.

Compound discrimination reversal. In contrast to performance on the EDS, performance on the discrimination reversal stage of the task was impaired by lesions of the orbital prefrontal cortex and not the lateral prefrontal cortex (Fig. 2A). ANOVA of the total errors to reach criterion on the discrimination reversal revealed a main effect of Lesion $\left(F_{(2,6)}=37.86 ; p<0.001\right)$. Moreover, although both lesioned and sham-operated control monkeys showed marked perseveration on this first reversal (EDSR) by continuing to respond to the previously correct exemplar for many trials, the extent of this perseveration was far greater in the orbital prefrontal lesioned monkeys than in the sham-operated controls or the lateral prefrontal lesioned monkeys.

If errors were classified as perseverative only until the monkeys had made their first correct response, then there were no significant differences across the three groups of monkeys $(F<1)$. However, it was clear from the data that the pattern of responding observed in those marmosets with selective lesions of the orbital prefrontal cortex was far more perseverative than that observed in both control and lateral prefrontal lesioned marmosets. Once control and lateral prefrontal lesioned monkeys had made a response away from the previously rewarded stimulus, they then continued to respond randomly until they shifted to the exemplar positively correlated with reward. By contrast, marmosets with lesions specific to the orbital prefrontal cortex returned to responding to the previously rewarded stimulus for many trials after making their first response away from that stimulus, although they had experienced reward for responding to the other stimulus (Fig. 3). Consequently, all errors within each half session (16 trials) were defined as perseverative if the monkey's performance across the 16 trials was significantly below chance (i.e., four or fewer correct responses). ANOVA of these data revealed that monkeys with lesions of the orbital prefrontal cortex made significantly more perseverative responses on the reversal, i.e., responses to the exemplar that had been rewarded previously, than either sham-operated controls or marmosets with lesions of the lateral prefrontal cortex $\left(F_{(2,6)}>100 ; p<0.0001\right)$.

To summarize, although lesions of the lateral prefrontal cortex increased selectively the number of errors made before attaining criterion on the EDS but not the IDS or reversal, lesions of the orbital prefrontal cortex increased selectively the number of errors made before attaining criterion on the reversal but not the IDSs or EDSs (Fig. 2A). An ANOVA of errors to criterion with 


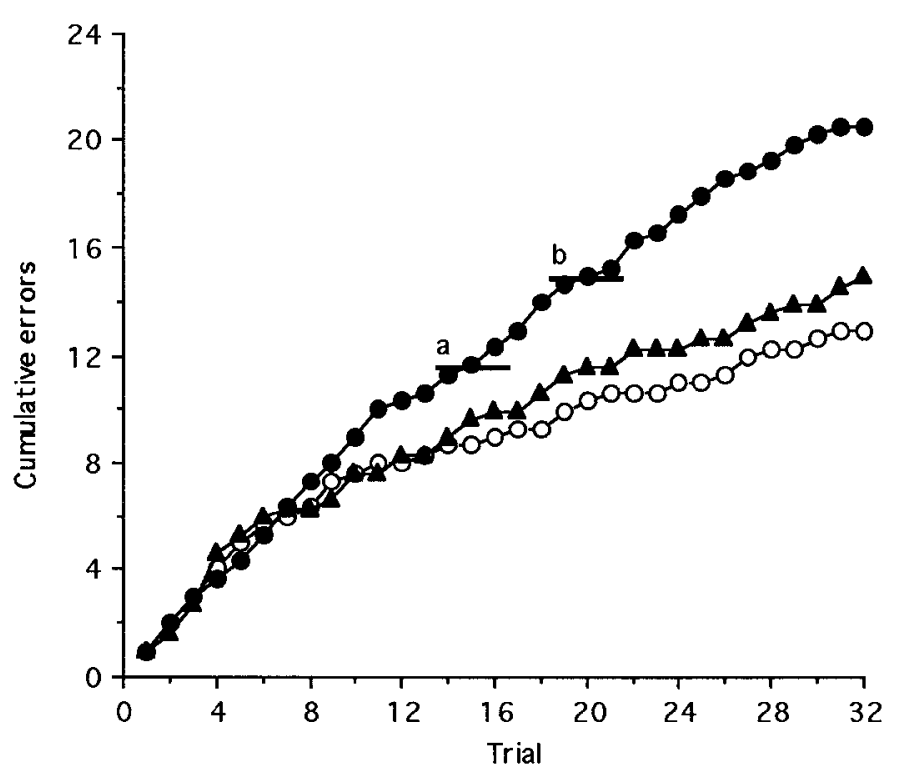

Figure 3. Mean cumulative errors made over the first session of the reversal (EDSR) by monkeys that received excitotoxic lesions of either the lateral (LAT) $(n=3)$ ( filled triangles) or orbital prefrontal cortex (ORB) $(n=3)$ (filled circles) or a sham operation $(n=3)$ (open circles). Horizontal line at $a$ and $b$ indicates the level of performance significantly different from chance $(p<0.05)$ on 16 trials, i.e., 12 or more incorrect responses, and 20 trials, i.e., 15 or more incorrect responses, respectively.

the factors of Lesion (lateral, orbital, and control) and Test (final IDS of the series of three that were presented postoperatively, EDS, and reversal) showed a main effect of Lesion $\left(F_{(2,6)}=12.72\right.$; $p<0.01)$ and an interaction of Lesion $\times$ Test $\left(F_{(4,12)}=18.92 ; p<\right.$ 0.001). Post hoc analysis of the simple main effects showed that there was a profound effect of lesioning on the $\operatorname{EDS}\left(F_{(2,18)}=\right.$ $23.78 ; p<0.001)$ and on the reversal $\left(F_{(2,18)}=25.57 ; p<0.001\right)$ but not on the IDS $\left(F_{(2,18)}=1.05\right)$.

Subsequent IDSs (IDS4 and IDS5) and reversals (IDS4R and $I D S 5 R)$. There was no effect of either lesion on acquisition of the subsequent series of novel discriminations or IDSs, which required the monkey to maintain attention toward the perceptual dimension that had been relevant at the immediately preceding EDS stage of the task (see Table 3 for mean \pm SEM). ANOVA of the errors to reach criterion for both discriminations revealed that there were no effects of Discrimination $(F<1)$ or Lesion $(F<1)$, and no Lesion $\times$ Discrimination interaction $(F<1)$. Neither was there any effect of either lesion on subsequent reversal learning. ANOVA of the total errors to reach criterion on the second (IDS4R) and third (IDS5R) discrimination reversals revealed that there were no effects of Reversal $(F<1)$ or Lesion $(F<1)$, and no Lesion $\times$ Reversal interaction $(F<1)$.

Second EDS and reversal (EDS2 and EDS2R). All monkeys took longer to acquire the discrimination requiring an EDS (EDS2) compared with the preceding discrimination requiring an IDS (IDS5), confirming that all monkeys had developed an attentional set to the second perceptual dimension and therefore were required to shift attentional set back to the dimension that was relevant at the start of the study (Fig. $2 B$ ). However, there were no significant differences between the three groups on this EDS2 (see Table 3 for mean \pm SEM). Similarly, there was no effect of either lesion on the compound discrimination reversal succeeding EDS2 $(F<1)$. ANOVA of the total errors to reach criterion on EDS2 and preceding IDS5 showed a main effect of Shift $\left(F_{(1,6)}=\right.$
109.09; $p<0.001)$, but no effect of Lesion $(F<1)$ and no Lesion $\times$ Shift interaction $(F<1)$.

\section{EXPERIMENT 2: INHIBITORY CONTROL VERSUS "ON-LINE" PROCESSING}

\section{Behavioral methods}

\section{Preoperative testing}

Simple visual discrimination and reversal. All monkeys $(n=9)$ were trained on a simple pattern discrimination (P1) consisting of a pair of green-dot patterns. A response to one of the patterns, $\mathrm{P} 1$, resulted in reward, whereas a response to the other pattern, $\mathrm{P} 2$, did not. All subjects received training on the pattern discrimination for 32 trials/day until they reached a criterion of $90 \%$ correct for three consecutive sessions. Once criterion was attained, on the following session the reward contingencies were reversed such that the pattern that had been negatively correlated with reward was now positively correlated with reward and vice versa; i.e., a response to $\mathrm{P} 2$ was now rewarded.

All monkeys were then allocated to one of three groups on the basis of their combined learning scores on the pattern discrimination and reversal. They then received infusions of the excitotoxin quinolinic acid into either the orbital (ORB group; $n=3$ ) or lateral (LAT group; $n=3$ ) regions of the prefrontal cortex, or they received sham surgery (control group; $n=3$ ).

\section{Postoperative testing}

Two weeks after surgery, all monkeys were tested for 32 trials/day on a series of visual discriminations, in which advancement to the next discrimination was contingent on reaching a performance level of $90 \%$ correct in two consecutive sessions. The full test design is presented in Table 1 (Experiment 2). A summary, however, is provided below.

(1) Retention of the pattern discrimination they had learned immediately before surgery.

(2) A series of three novel compound discriminations or IDSs (IDS1, IDS2, IDS3). All monkeys were assessed on their ability to learn a compound visual discrimination comprising two novel dimensions: black lines superimposed over blue-filled shapes (Fig. 1c). To succeed, a monkey had to respond to one of the two exemplars from the relevant dimension, ignoring both exemplars from the irrelevant dimension. Initially, six monkeys were presented with "shape" as the relevant dimension, as illustrated in Figure 1, and the remaining three monkeys were presented with "lines" as the relevant dimension.

(3) Two novel compound discriminations or IDSs and subsequent reversals (IDS4, IDS4R, IDS5, IDS5R). In the discriminations requiring an IDS, the monkey was required to learn which of two novel exemplars from the previously relevant dimension was positively correlated with reward. In the reversals, the exemplar that had been previously negatively correlated with reward in discriminations IDS4 and IDS5, respectively, became positively correlated with reward and vice versa. The exemplars from the irrelevant dimension remained uncorrelated with reward.

This extended series of ID shifts and reversals was used in the present experiment to ensure that all monkeys had "tuned in" to one specific perceptual dimension, thereby enabling the acquisition of an attentional set to be assessed fully.

(4) A probe test. After completion of the reversal of the fifth IDS (IDS5R), the exemplars from the irrelevant dimension were replaced with two novel exemplars, whereas the exemplars from the relevant dimension and the reward contingencies remained 


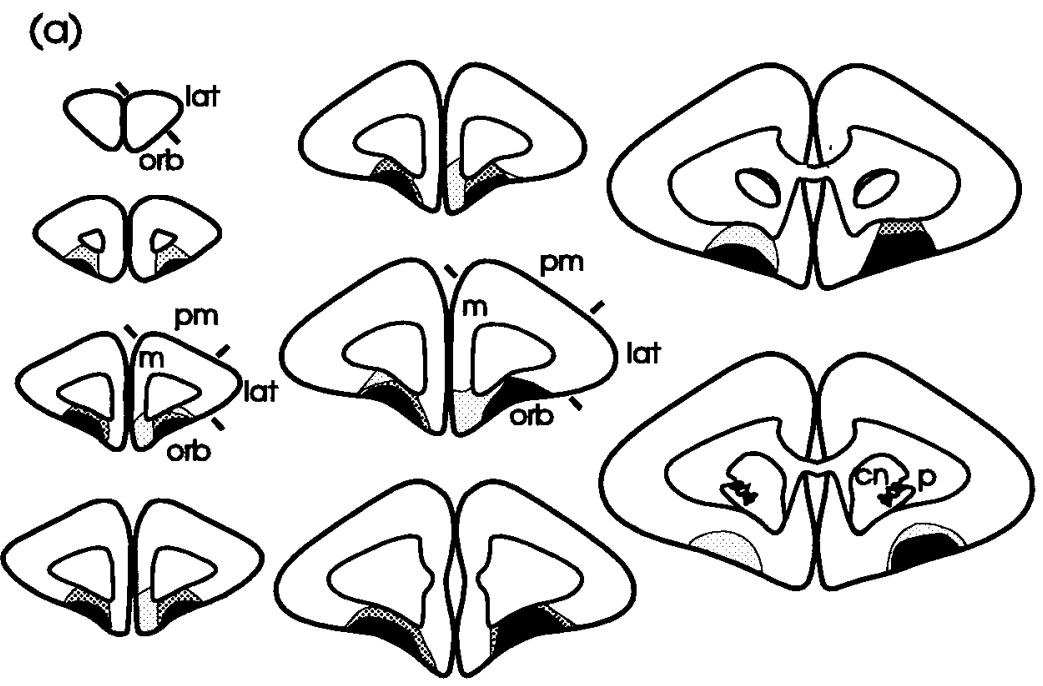

Figure 4. Schematic diagrams of a series of coronal sections through the frontal lobe illustrating the site of the lesion of the orbital $(a)$ and lateral $(b)$ prefrontal cortices. The three different types of shading represent the area of tissue that was damaged in all three marmosets (black shading), in two of the three marmosets (dark stippling), and in one marmoset only (pale stippling) after an orbital $(a)$ or a lateral lesion $(b)$. Orbital prefrontal cortex corresponds to areas 10-13 (marked $o r b$ on the sections), and lateral prefrontal cortex corresponds to area 9 (marked lat on the sections), as defined by Brodmann (1909) in his description of the frontal cortex in the marmoset. $m$, Medial prefrontal cortex; $p m$, premotor cortex; $c n$, caudate nucleus; $p$, putamen).

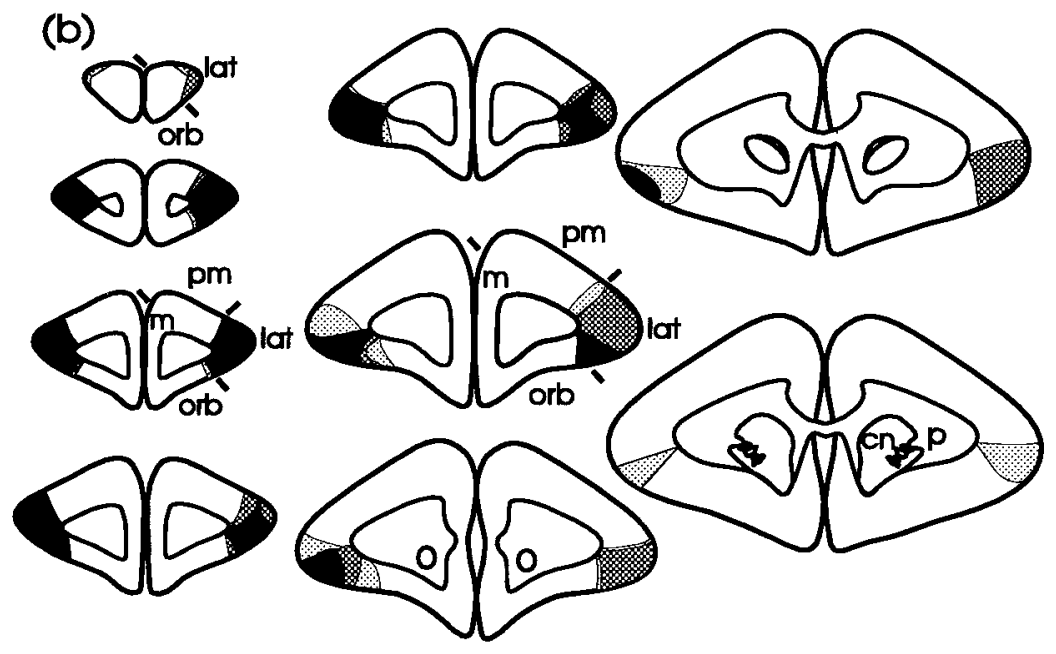

the same; i.e., the exemplar from the relevant dimension that had been previously correlated with reward continued to be correlated with reward. Once the monkeys had reattained criterion they were returned to the original compound discrimination before the next stage.

(5) An EDS. A new compound discrimination in which one of the two novel exemplars from the previously irrelevant dimension became positively correlated with reward, thus requiring a shift of attentional set from one perceptual dimension of the compound stimulus to another (Fig. 1d).

(6) A compound discrimination reversal (EDSR). The exemplar that had been previously negatively correlated with reward in discrimination EDS became positively correlated with reward and vice versa (Fig. 1e). The exemplars from the irrelevant dimension remained uncorrelated with reward.

Again, the number of errors that were made before reaching criterion were recorded for each discrimination.

\section{Statistical methods}

All behavioral data were analyzed using the CLR ANOVA statistical package (Clear Lake Research). Whenever the distribution of these variables violated the assumptions made for the ANOVA, an appropriate transformation was used. Planned comparisons were made using simple main effects.

\section{Results}

\section{Histological assessment of lesion}

Examination of the cresyl fast violet-stained sections of the orbital and lateral prefrontal lesioned marmosets revealed that both discrete prefrontal regions had been selectively destroyed by the excitotoxin (Figs. 4 and 5). In all three marmosets of the orbital prefrontal lesioned group, there was extensive damage, bilaterally, throughout the orbital region of prefrontal cortex (Figs. 4a, Fig. $5 a, b)$. The most anterior limit of the area of cell loss was just caudal to the frontal pole, whereas the posterior limit extended just rostral to the putamen ( $\mathrm{pm}$ ) (Fig. $4 a$ ). In one of the marmosets, damage in the orbital region was confined to the supragranular layers and layer IV and did not extend into the infragranular layers. The cortex was spared in the adjacent lateral (lat) and medial $(m)$ prefrontal regions (Fig. $4 a$ ).

In the lateral prefrontal lesioned group, in two of the three lesioned marmosets there was a near total loss of cells, bilaterally, in the lateral prefrontal cortex extending from the frontal pole, anteriorly, to the level of the anterior limit of the caudate nucleus $(c n)$, posteriorly (Figs. $4 b, 5 c, d$ ). However, in the third marmoset, although damage to the right side of the lateral prefrontal cortex was similar in extent to that seen bilaterally in the other two lateral prefrontal lesioned marmosets, damage to the left side was 

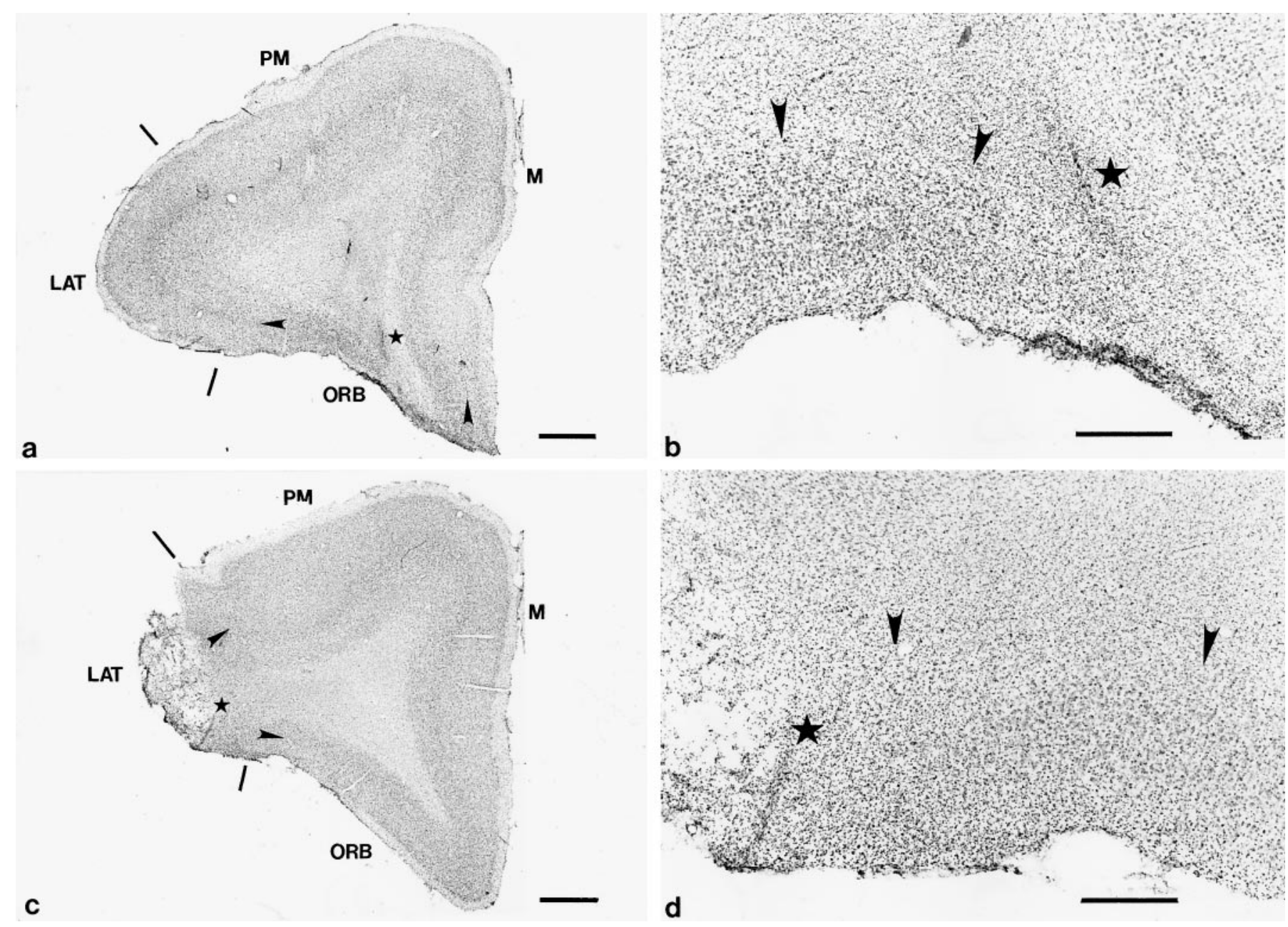

Figure 5. Low $(a, c)$ and high $(b, d)$ power photomicrographs of cresyl violet-stained coronal sections through an intermediate level of the frontal pole taken from a representative marmoset from both the orbital $(a, b)$ and lateral $(c, d)$ lesioned groups. The extensive cell loss in the orbital $(O R B)$ prefrontal cortex in $a$ is in stark contrast to the dense layering of neurons seen in the same region in $c$. Similarly, the almost total loss of neurons in the lateral prefrontal cortex $(L A T)$ in $c$ is in marked contrast to the dense layering of neurons seen in the same region in $a$. The stars mark the same locations in each pair of low and high power photomicrographs (e.g., $a$ and $b, c$ and $d$ ); large arrowheads in $b$ and $d$ mark the boundaries between the cortex and white matter; small arrowheads in $a$ mark the lateral and medial boundaries of the orbital prefrontal lesion; small arrowheads in $c$ mark the medial and dorsal boundaries of the lateral prefrontal lesion. Scale bars: $a, c, 1 \mathrm{~mm} ; b, d, 400 \mu \mathrm{m}$. $M$, Medial prefrontal cortex; $P M$, premotor cortex.

less extensive (Fig. 4b). Although there was a total loss of cells within the left lateral prefrontal cortex at the frontal pole, there was some sparing of cells in the supragranular layers of the lateral prefrontal cortex more posteriorly. In all three lateral prefrontal lesioned marmosets, the cortex in the adjacent premotor areas $(\mathrm{pm})$ and orbital areas (orb) was spared (Fig. $4 b$ ).

\section{Behavioral effects}

Retention test. Preoperatively, the monkeys that were scheduled to receive either quinolinic acid or phosphate buffer vehicle into the lateral or orbital regions of prefrontal cortex did not differ in their ability to learn a simple visual pattern discrimination and reversal $(F<1)$ (see Table 4 for mean \pm SEM).

Postoperatively, although there was a trend for the orbital prefrontal lesioned monkeys to make fewer errors than either controls or lateral prefrontal lesioned marmosets in regaining criterion on the retention test (see Table 4 for mean \pm SEM), this did not reach the $5 \%$ level of significance $\left(F_{(2,6)}=3.59 ; p=0.09\right)$.

Acquisition of a novel attentional set. There was no effect of either lesion on the acquisition of the first novel compound visual
Table 4. Mean scores $( \pm$ SEM) for Experiment 2

Mean number of errors to criterion \pm SEM

\begin{tabular}{lccc}
\cline { 2 - 4 } Discrimination & Control & LAT lesion & ORB lesion \\
\hline All preoperative discriminations & $174.0 \pm 55.0$ & $143.8 \pm 46.6$ & $156.2 \pm 31.1$ \\
Retention test (P2) & $8.7 \pm 2.5$ & $12.3 \pm 4.0$ & $2.7 \pm 1.8$ \\
Intradimensional shift 1 (IDS1) & $34.3 \pm 4.7$ & $34.0 \pm 11.2$ & $37.3 \pm 9.2$ \\
Intradimensional shift 2 (IDS2) & $60.0 \pm 12.3$ & $51.7 \pm 7.8$ & $50.3 \pm 9.6$ \\
Intradimensional shift 3 (IDS3) & $18.3 \pm 2.2$ & $20.0 \pm 3.2$ & $20.7 \pm 2.5$ \\
Intradimensional shift 4 (IDS4) & $5.0 \pm 3.9$ & $0.0 \pm 0.0$ & $3.0 \pm 3.0$ \\
Compound reversal 1 (IDS4R) & $25.0 \pm 4.6$ & $28.0 \pm 5.5$ & $47.3 \pm 5.5^{*}$ \\
Intradimensional shift 5 (IDS5) & $3.7 \pm 2.3$ & $2.7 \pm 1.6$ & $4.7 \pm 3.6$ \\
Compound reversal 2 (IDS5R) & $9.7 \pm 2.2$ & $9.0 \pm 2.5$ & $10.3 \pm 1.6$ \\
Extradimensional shift (EDS) & $18.3 \pm 4.1$ & $40.7 \pm 3.6^{*}$ & $22.3 \pm 2.5$ \\
Compound reversal 3 (EDS1R) & $14.3 \pm 3.3$ & $14.3 \pm 2.2$ & $15.7 \pm 2.5$
\end{tabular}

$* p<0.001$. 
Figure 6. Mean number of errors $( \pm$ SEM) made by shamoperated controls (open bars) $(n=3)$, lateral prefrontal lesioned marmosets (pale hatched bars) $(n=3)$, and orbital prefrontal lesioned marmosets (dark hatched bars) $(n=3)$ to reach criterion on a series of five IDSs.

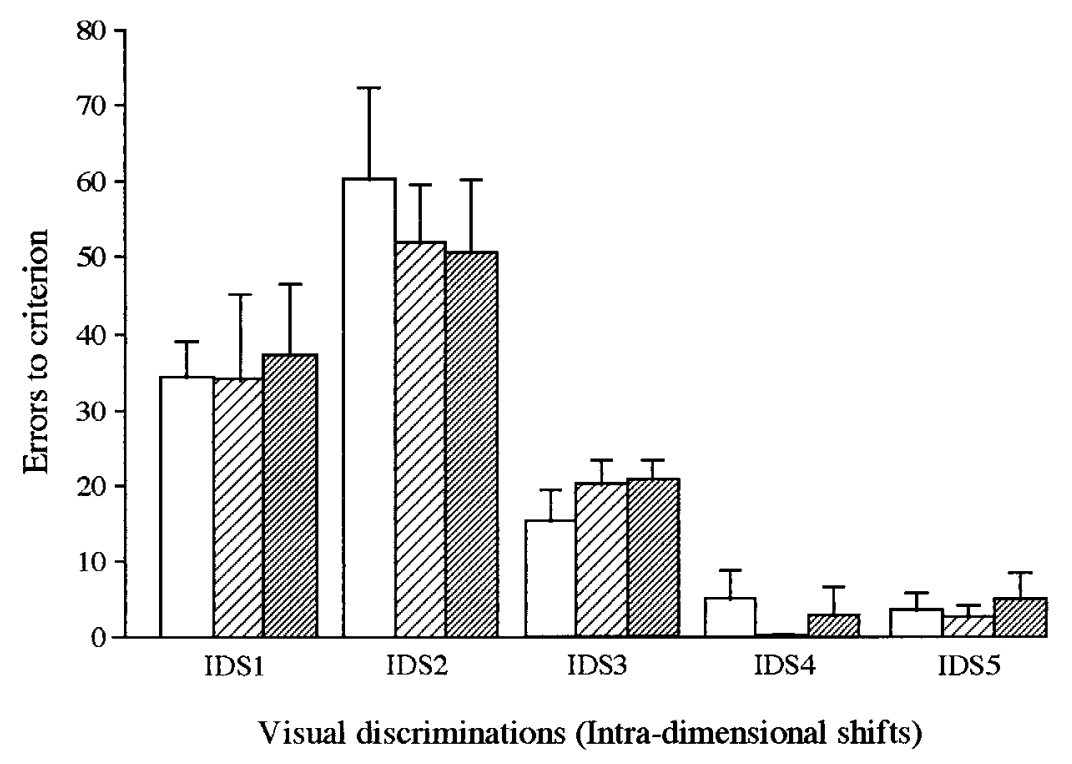

tion $\left(F_{(2,6)}=18.34 ; p<0.01\right)$. Further analysis of the simple main effects showed that the orbital prefrontal lesioned marmosets made significantly more perseverative errors than controls $\left(F_{(2,12)}\right.$ $=56.64 ; p<0.001)$, but did not differ from controls in the number of nonperseverative errors $(F<1)$.

Probe test. The introduction of novel exemplars of the irrelevant dimension had no effect on performance in control, lateral prefrontal, or orbital prefrontal lesioned monkeys; in all three groups the monkeys continued to respond to the previously correct exemplar at the $90 \%$ correct criterion level. A comparison of the errors made on the previous test session when all monkeys were performing at the $90 \%$ criterion level revealed no effect of the probe $(F<1)$ or the lesion $(F<1)$, and no interaction of the lesion with the probe $(F<1)$.

$E D S$ and reversal. All monkeys took longer to acquire the discrimination requiring an EDS compared with the preceding discrimination requiring an IDS, again confirming that all monkeys had indeed developed an attentional set and therefore, were required to shift attentional set at the EDS stage of the test. In agreement with Experiment 1, although the performance of monkeys with lesions of the lateral prefrontal cortex was equivalent to both controls and monkeys with lesions of the orbital prefrontal cortex on a discrimination requiring an IDS, their performance was significantly inferior to both of the other groups on the discrimination requiring an EDS. Monkeys with lesions of the lateral prefrontal cortex made nearly three times as many errors as either orbital prefrontal lesioned monkeys or shamoperated controls (Table 4). ANOVA of the total errors to reach criterion on the EDS and preceding IDS (IDS5) showed a main effect of Lesion $\left(F_{(2,6)}=5.87 ; p<0.05\right)$, a main effect of Shift $\left(F_{(1,6)}=343.70 ; p<0.001\right)$, and a significant Lesion $\times$ Shift interaction $\left(F_{(2,6)}=34.98 ; p<0.001\right)$. Further analysis of the simple main effects showed that the lateral prefrontal lesioned marmosets were selectively impaired at the ED shift stage of the task $\left(F_{(2,8)}=20.67 ; p<0.001\right)$. Moreover, there was evidence in this experiment that the impairment in the monkeys with lateral prefrontal lesions may have been the result of perseveration to the previously relevant dimension. As in Experiment 1, two of the three sham-operated monkeys and all three monkeys with orbital prefrontal lesions responded repeatedly to one of the exemplars (five or six consecutive responses; $p<0.05$ ) from the previously nonperseverative) $\left(F_{(1,6)}=318.50 ; p<0.001\right)$, Lesion $\left(F_{(2,6)}=\right.$ 41.13; $p<0.001)$, and a significant Lesion $\times$ Error type interac- 
relevant dimension in the first session of the EDS. A similar pattern of responding was seen in all three monkeys with lateral prefrontal lesions, except that they made seven or eight consecutive responses $(p<0.01)$ and were more likely to repeat this series of responses. Monkeys were given a score of 1 for every run of five or six responses and 2 for every run of seven or eight responses, and a comparison of their performance on the immediately preceding IDS and the subsequent EDS showed that all monkeys responded to the previously relevant dimension at the EDS stage (main effect of Shift: $F_{(1,6)}=54 ; p<0.003$ ) and there was a nonsignificant trend for the lesioned monkeys to exhibit this behavior more than controls or monkeys with orbital prefrontal lesions (Lesion $\times$ Shift interaction: $F_{(2,6)}=4.5 ; p=0.064$ ). By contrast, there was no effect of either lesion on the subsequent reversal of this visual discrimination requiring an ED shift $(F<1)$.

To summarize, neither lesion of the lateral or orbital regions of the prefrontal cortex impaired the ability of the marmoset to acquire an attentional set of the relevant perceptual dimension. However, in agreement with experiment 1 , lesions of the orbital prefrontal cortex impaired performance selectively on the first but not subsequent reversals, whereas lesions of the lateral prefrontal cortex impaired performance selectively on the EDS.

\section{DISCUSSION}

The present findings demonstrate that although lesions of the orbital but not the lateral prefrontal cortex impaired the ability of marmosets to reverse a stimulus-reward association within a particular perceptual dimension, (e.g., shapes), lesions of the lateral rather than the orbital prefrontal cortex impaired the ability to shift an attentional set from one perceptual dimension to another (e.g., shapes to lines). These impairments were restricted to the first occasion that such shifts in responding were required. Performance on subsequent reversals of stimulus-reward associations or on an additional shift of an attentional set was unaffected. The finding that neither lesion disrupted the ability of marmosets to perform compound visual discriminations per se or to develop (Exp. 2) or maintain (Exp. 1) an attentional set toward one particular perceptual dimension highlights further the specificity of the deficit to one of a disruption of inhibitory control mechanisms.

\section{Inhibitory control versus on-line processing}

Traditionally, impairments in inhibitory control shown by patients with damage to the prefrontal cortex on tests such as the Wisconsin Card Sort Test (WCST) have been accounted for in terms of deficits in inhibiting an "attentional set" (Milner, 1964; Mishkin, 1964; Cicerone and Lazur, 1983). However, more recently Goldman-Rakic (1987) has attempted to incorporate such impairments into a working memory account of prefrontal function, suggesting that the impairments in inhibitory control may reflect an underlying deficit in the ability to hold information on line in working memory (Goldman-Rakic, 1987). The results of the present study cannot be easily accommodated by this particular working memory theory of prefrontal function, because all the discriminations could be solved using short-term memory but only those involving inhibition of a previously acquired response were impaired. It can be argued that the memory load needed to perform an IDS was smaller than that required to perform an EDS in Experiment 1, because the monkeys had already acquired an attentional set to a particular perceptual dimension. Therefore, unlike the EDS, they could ignore a proportion of the available stimulus cues, i.e., exemplars from the irrelevant dimension. However, because the lesioned monkeys in Experiment 2 had no previous experience with the compound stimuli, it follows that the first discrimination after surgery was performed by all monkeys before acquiring an attentional set, and hence the working memory requirements were equivalent to those of an EDS. A more parsimonious explanation is that the impairments were attributable to a loss of inhibitory control at two different levels of cognitive processing: affective processing (after lesions of the orbital prefrontal cortex) and attentional selection (after lesions of the lateral prefrontal cortex). That neither lesion disrupted the ability to learn compound discriminations per se or to acquire an attentional set demonstrates further the disinhibitory nature of these deficits, thus ruling out more general roles for these regions in processes of response selection based on emotional and higherorder attentional factors.

Whether other regions of the prefrontal cortex are involved more generally in response selection based on emotional and higher-order attentional factors is unclear. Certainly lesions involving areas 13, 14, and 32 on the ventromedial and medial surfaces in macaque monkeys impair the ability to associate reward with particular visual stimuli (Iversen and Mishkin, 1970; Gaffan and Murray, 1990; Gaffan et al., 1993), a deficit similar to that seen after damage to the amygdala (Gaffan and Murray, 1990), with which these regions of prefrontal cortex are heavily interconnected (Jones and Powell, 1970; Nauta, 1971; Amaral et al., 1992). Whether the prefrontal cortex is also involved in the ability to acquire an attentional set is less clear. A study by Iversen and Mishkin (1970) did show that lesions restricted to areas 13 and 14 on the orbital surface abolished the improvement in performance that is normally seen across a series of visual discrimination reversals, an impairment that could have reflected the failure of such an attentional mechanism (Mackintosh and Little, 1969). Certainly, these regions are interconnected with the inferotemporal cortex (Ungerleider et al., 1989), which has been implicated specifically in visual selective attention (Butter, 1969; Gross et al., 1971).

\section{The importance of novelty}

The finding that the impairments in inhibitory control were present only on the first occasion that shifts of responding were required suggests that the prefrontal cortex provides inhibitory control particularly in novel situations. Lesioning the orbital prefrontal cortex only impaired performance on the first discrimination reversal, regardless of whether the first reversal was presented before (Exp. 2) or after (Exp. 1) the EDS stage of the task. This is consistent with previous results in which the perseverative responding induced by lesions of the orbitofrontal cortex in rhesus monkeys was restricted to only the first reversal of a series of reversals (Iversen and Mishkin, 1970). Similarly, lesions of the lateral region of prefrontal cortex in the present study only impaired performance on the first discrimination requiring an EDS. There was no impairment on the second EDS, which required monkeys to re-engage a previously established attentional set. It might be argued that this second EDS would be a relatively insensitive test, because not only have the monkeys already been exposed to an EDS once, but also they are shifting back to a dimension that has been relevant in the recent past. However, this ability to re-engage a previously relevant attentional set has been shown to be disrupted by 6-hydroxydopamine lesions of the entire head and body of the caudate nucleus, whereas the same lesion does not appear to disrupt the first EDS (Roberts et al., 1994). 
This double dissociation indicates that the restriction of the shifting deficit to the first EDS in the present study is not caused by task insensitivity. Rather, these findings suggest that the lateral and orbital prefrontal cortex may no longer be necessary for the subsequent application of algorithms or rules underlying reversal learning and attentional set-shifting within the context of a specific task when these have already been established by previous training.

\section{Functional organization within the prefrontal cortex}

This double dissociation between the behavioral effects of lesions of the lateral and orbital prefrontal cortex in the marmoset on discrimination reversal learning and attentional set-shifting provides new insight into the functional organization of the prefrontal cortex. It suggests that distinct regions of the prefrontal cortex carry out independent but complementary forms of cognitive processing of complex visual stimuli in changing environmental circumstances. Thus, regions within the orbital prefrontal cortex in marmosets enable the rapid reversal of affective associations for specific visual stimuli, whereas the higher-order shifting of attention between supraordinate features of visual stimuli, such as their perceptual dimensions, is mediated by regions within the lateral prefrontal cortex. These findings run contrary to traditional theories of prefrontal function in which inhibitory control has been associated primarily with orbital prefrontal cortex in monkeys (Mishkin, 1964; Fuster, 1985). They suggest instead that inhibitory control may be operating across a number of distinct functional regions within the prefrontal cortex. Additional support for this hypothesis comes from ablation (Diamond and Goldman-Rakic, 1989) and electrophysiological recording (Funahashi et al., 1993) studies in rhesus monkeys, which demonstrate that inhibitory control mechanisms operate together with on-line processing within the dorsolateral prefrontal cortex to control performance on the spatial delayed response task. These findings also run contrary to original theories of selective attention and discrimination learning in animals (Sutherland and Mackintosh, 1971), which suggested that there was a hierarchical relationship between the selection of an appropriate perceptual dimension and the affective "tagging" of particular stimuli within that dimension as rewarded or not. The double dissociation between the behavioral effects of lateral and orbital lesions on set-shifting and reversal learning in the present study suggests instead that these processes occur in parallel. Finally, these findings also provide an explanation for the apparent discrepancy between human and nonhuman primate studies with respect to the anatomical locus of inhibitory control. Thus, the impairment in shifting categories on the WCST that has been associated with damage to dorsolateral but not orbital prefrontal cortex in humans (Milner, 1964; Eslinger and Damasio, 1985; Shallice and Burgess, 1991) is similar to the impairment in shifting an attentional set described in the present study that is associated with damage to the lateral rather than orbital prefrontal cortex in marmosets.

Two other modes of organization have been postulated to occur within the prefrontal cortex in addition to the one suggested here. The first, sensory-specific processing, is based on findings from electrophysiological (Wilson et al., 1993) and functional neuroimaging (Courtney et al., 1996) studies and suggests that the prefrontal cortex is involved in the general process of holding representations of stimulus information on-line, with independent analysis of visual and spatial information in adjacent regions. The second, based on evidence from functional neuro- imaging and ablation studies, suggests that neighboring regions of dorsal prefrontal cortex make distinct, possibly hierarchical, contributions to memory that differ in terms of the nature of the processing of the information that is held in posterior association cortex (e.g., simple retrieval vs monitoring of information) (twostage theory of Petrides, 1996; Owen et al., 1996). It is unclear which of these positions is best supported by the present data, which in some respects are compatible with either. Thus, we have shown that mechanisms of response inhibition are present in distinct cognitive domains for affective as well as higher-order processing of visual stimuli. This organization is not inconsistent with the existence of sensory-specific domains, although the nature of the impairments seen in the present study are inconsistent with a generalized impairment in on-line processing. Indeed, they support the recent findings of Rushworth et al. (1997) that emphasize the importance of the prefrontal cortex in response selection beyond those situations in which stimuli must be held on-line. The present findings are also consistent with the twostage account of Petrides (1996) in that they provide evidence for functionally distinct processing domains. They differ from this account, however, in arguing for the existence of parallel processing of affective and higher-order information in different regions of the prefrontal cortex rather than hierarchical processing proposed in the two-stage account. Indeed, in agreement with our own thesis these results have been taken to support the role of different regions of prefrontal cortex in the selection and control of action based on lower-order and higher-order rules (Wise et al., 1996).

\section{REFERENCES}

Amaral DG, Price JL, Pitkanen A, Carmichael ST (1992) The primate amygdala. In: The amygdala (Aggleton JP, ed), pp 1-66. New York: Wiley.

Brodmann K (1909) Vergleichende lokalisationslehre der großhirnrinde in ihren prinzipierdargestellt auf grund des zellenbaues. Leipzig: Verlag Johan Ambrosius Barth.

Butter CM (1969) Impairments in selective attention to visual stimuli in monkeys with inferotemporal and lateral striate lesions. Brain Res 12:374-383.

Cicerone KD, Lazar RM (1983) Effects of frontal lobe lesions on hypothesis sampling during concept formation. Neuropsychologia 21:513-524.

Courtney SM, Ungerleider LG, Keil K, Haxby JV (1996) Object and spatial visual working memory activate separate neural systems in human cortex. Cereb Cortex 6:39-49.

Diamond A (1990) Developmental time course in human infants and infant monkeys, and the neural bases of inhibitory control in reaching. Ann NY Acad Sci 608:637-676.

Diamond A, Goldman-Rakic PS (1989) Comparison of human infants and rhesus monkeys on Piaget's AB task: evidence for dependence on dorsolateral prefrontal cortex. Exp Brain Res 74:24-40.

Dias R, Robbins TW, Roberts AC (1996a) Dissociation in prefrontal cortex of affective and attentional shifts. Nature 380:69-72.

Dias R, Robbins TW, Roberts AC (1996b) Primate analogue of the Wisconsin Card Sort Test: effects of excitotoxic lesions of the prefrontal cortex in the marmoset. Behav Neurosci 110:872-886.

Eslinger PJ, Damasio AR (1985) Severe disturbance of higher cognition after bilateral frontal lobe ablation: patient E. V. R. Neurology 35:1731-1741.

Funahashi S, Bruce CJ, Goldman-Rakic PS (1993) Dorsolateral prefrontal lesions and oculomotor delayed response performance: evidence for mnemonic "scotomas." J Neurosci 13:1479-1497.

Fuster JM (1985) The prefrontal cortex and temporal integration. In: Cerebral cortex, Vol 4 (Jones EG, Peters A, eds), pp 151-177. New York: Plenum.

Fuster JM (1989) The prefrontal cortex. New York: Raven.

Gaffan D, Murray EA (1990) Amygdala interaction with the mediodorsal nucleus of the thalamus and the ventromedial prefrontal cortex in 
stimulus-reward associative learning in the monkey. J Neurosci 10:3479-3493.

Gaffan D, Murray EA, Fabre-Thorpe M (1993) Amygdala-prefrontal interaction in stimulus-reward associative learning. Eur J Neurosci 5:968-975.

Goldman-Rakic PS (1987) Circuitry of primate prefrontal cortex and regulation of behaviour by representational memory. In: Handbook of physiology, the nervous system, higher functions of the brain, Vol V (Plum F, Mountcastle V, eds), pp 373-417. Bethesda, MD: American Physiological Society.

Gross CG, Cowey A, Manning FJ (1971) Further analysis of visual discrimination deficits following foveal prestriate and inferotemporal lesions in monkeys. J Comp Physiol Psychol 76:1-7.

Iversen SD, Mishkin M (1970) Perseverative interference in monkeys following selective lesions of the inferior prefrontal convexity. Exp Brain Res 11:376-386.

Jones EG, Powell TPS (1970) An anatomical study of converging sensory pathways within the cerebral cortex of the monkey. Brain 93:793-820.

Knight RT, Grabowecky M (1995) Escape from linear time: prefrontal cortex and conscious experience. In: The cognitive neurosciences (Gazzaniga MS, ed), pp 1357-1372. Cambridge: MIT.

Mackintosh NJ, Little L (1969) Selective attention and response strategies as factors in serial reversal learning. Can J Psychol 23:335-346.

Milner B (1964) Some effects of frontal lobectomy in man. In: The frontal granular cortex and behavior (Warren JM, Akert K, eds), pp 313-334. New York: McGraw-Hill.

Mishkin M (1964) Perseveration of central sets after frontal lesions in man. In: The frontal granular cortex and behavior (Warren JM, Akert K, eds), pp 219-294. New York: McGraw-Hill.

Nauta WJH (1971) The problem of the frontal lobe; a reinterpretation. J Psychiat Res 8:167-187.

Owen AM, Roberts AC, Hodges JR, Summers BA, Polkey CE, Robbins TW (1993) Contrasting mechanisms of impaired attentional setshifting in patients with frontal lobe damage or Parkinson's disease. Brain 116:1159-1175.

Owen AM, Evans AC, Petrides M (1996) Evidence for a two-stage model of spatial working memory processing within the lateral frontal cortex: a positron emission tomography study. Cereb Cortex 6:31-38.

Petrides M (1996) Specialized systems for the processing of mnemonic information within the primate frontal cortex. In: Cognitive and executive functions of the prefrontal cortex (Roberts AC, Robbins TW, Weiskrantz L, eds). Philos Trans R Soc Lond [Biol] 351:1387-1527.

Roberts AC, Robbins TW, Everitt BJ (1988) The effects of intradimensional and extradimensional shifts on visual discrimination learning in humans and non-human primates. Q J Exp Psychol 40:321-341.

Roberts AC, Robbins TW, Everitt BJ, Muir JL (1992) A specific form of cognitive rigidity following excitotoxic lesions of the basal forebrain in the marmoset. Neuroscience 47:251-264.

Roberts AC, Collins P, Wilkinson LS, Everitt BJ, Robbins TW (1994) Primate analogue of the Wisconsin Card Sort Test (WCST): a behavioural and microdialysis study of striatal dopamine function in the marmoset. Soc Neurosci Abstr 20:357.

Rolls ET, Hornak J, Wade D, McGrath J (1994) Emotion-related learning in patients with social and emotional changes associated with frontal lobe damage. J Neurol Neurosurg Psychiatry 57:1518-1524.

Rushworth MFS, Nixon PD, Eacott MJ, Passingham RE (1997) Ventral prefrontal cortex is not essential for working memory. J Neurosci 17:4829-4838.

Shallice T, Burgess PW (1991) Deficits in strategy application following frontal lobe damage in man. Brain 114:727-741.

Shallice T, Burgess PW (1993) Supervisory control of action and thought selection. In: Attention, selection, awarement and control (Baddeley A, Weiskrantz L, eds), pp 171-187. Oxford: Clarendon.

Sutherland NS, Mackintosh NJ (1971) Mechanisms of animal discrimination learning. New York: Academic.

Ungerleider LG, Gaffan D, Pelak VS (1989) Projections from inferior temporal cortex to prefrontal cortex via the uncinate fascicle in rhesus monkeys. Exp Brain Res 76:473-484.

Wilson FAW, O'Scalaide P, Goldman-Rakic PS (1993) Dissociation of object and spatial processing domains in primate prefrontal cortex. Science 260:1955-1958.

Wise SP, Murray EA, Gerfen CR (1996) The frontal cortex-basal ganglia system in primates. Crit Rev Neurobiol 10:317-35. 